\title{
Förderung ambulanter Leistungen
}

\section{Michael Stamm}

Dr. med., Executive MBA, Facharzt für Anästhesiologie und Intensivmedizin, medizinischer Leiter und Geschäftsführer der Operationszentrum Burgdorf AG

\author{
Jetzt und in Zukunft beschäftigen fünf grosse Problemkreise das Schweizer \\ Gesundheitswesen: laufende Zunahme der Gesundheitskosten, Abnahme der \\ Entgeltung stationär erbrachter Leistungen, grosser Investitionsbedarf, hohe Kom- \\ plexität der Leistungserstellung, Fachkräftemangel. Mehr ambulant statt statio- \\ när erbrachte Leistungen sind ein Weg, diesen Herausforderungen wirkungsvoll \\ zu begegnen.
}

Encourager les prestations ambulatoires pour répondre aux défis du système de santé?

Du point de vue médical, économique et social, il est préférable d'opter pour des traitements et des opérations ambulatoires en lieu et place de I'hospitalisation traditionnelle, si cela est médicalement justifié. Cette solution permet en effet de diminuer la hausse des coûts dans le système de santé; de continuer à fournir les prestations médicales de façon rentable malgré une indemnisation dégressive dans le système DRG; de mieux différencier les investissements dans les établissements médicaux tout en diminuant les coûts; de réduire la complexité des prestations et d'améliorer leur qualité; et enfin, de remédier à la pénurie de personnel qualifié de manière effective.

L'indemnisation des prestations constitue le levier de toutes ces améliorations: elle devrait avoir lieu indépendamment du type de prestation (ambulatoire ou hospitalière); I'indemnisation des frais supplémentaires qu'implique une hospitalisation traditionnelle devrait être proportionnée; I'indemnisation des prestations fournies de manière ambulatoire devrait être améliorée, aussi bien de façon relative par rapport aux prestations hospitalières équivalentes mais également de façon absolue; et les prestations additionnelles devraient également être indemnisées dans le secteur ambulatoire. En encourageant les traitements et les opérations ambulatoires, l'ensemble des acteurs de la santé seraient incités à améliorer le développement du système et à stimuler l'innovation.

Lesen Sie hierzu auch den Kommentar von Anna Sax auf Seite 407 .

Die Literaturangaben finden sich unter www. saez.ch $\rightarrow$ Aktuelle Ausgabe oder $\rightarrow$ Archiv $\rightarrow 2015$ $\rightarrow 11$
Jahren 2000-2012 um 12,8\%. Der Anteil der über 65-Jährigen (nicht mehr Erwerbstätige, Hauptkostenverursacher) stieg in der gleichen Zeit um 26,1\% [3]. Die Differenz dieser beiden Bevölkerungsanteile beträgt 13,3\%. Sie alleine erklärt nicht, wieso die Gesundheitsausgaben stärker als das BIP steigen. Auch der oft zitierte "medizinische Fortschritt» als Kostentreiber muss hinterfragt werden. Um uns herum wird Dank Fortschritt alles billiger. Wieso sollte dies nicht auch im Gesundheitswesen so sein?

Der Umstand, dass die Kosten im Gesundheitswesen stärker als das BIP ansteigen, deutet darauf hin, dass Ressourcen verschwendet werden. Tatsächlich gibt es im Schweizer Gesundheitswesen finanzielle Fehlanreize [4], die zu unnötigen teuren stationären Behandlungen führen. Stationäre Behandlungen werden nach SwissDRG unverhältnismässig besser abgegolten als die genau gleiche Leistung ambulant erbracht nach TARMED (Beispiel Krampfadern-Operation: 3-mal besser). Bei zusatzversicherten Patienten ist dies besonders krass ausgeprägt (Beispiel Krampfadern-Operation: 8-mal besser) [5]. Die Gesundheitsausgaben 2012 stiegen gegenüber dem Vorjahr denn auch insbesondere in den Spitälern $(+9,8 \%)$ [6]. Die Folgen sind unnötige Ausgaben, Fehlallokation von Personal, Knowhow und Geld, sowie befangene Leistungserbringer, die aufgrund der Fehlanreize nicht nur im Sinne der Patienten entscheiden.

Das Schweizer Gesundheitswesen steht vor der Herausforderung, die Ausgaben nicht nur zu senken, sondern die personellen und finanziellen Ressourcen effektiver (die richtigen Dinge tun) einzusetzen.

Eine wichtige Handlungsoption ist die Beseitigung der finanziellen Fehlanreize, die zugunsten stationär erbrachter Leistungen bestehen. Die Einführung einer monistischen Abgeltung erscheint hierfür besonders prüfenswert: Medizinische Leistungen sollen unab- 
Tabelle 1: Durchschnittliche Baserate Akutsomatik exkl. Spezialkliniken, Universitäts- und Kinderspitäler (in CHF). EG HSK = Einkaufsgemeinschaft HELSANA SANITAS KPT.

\begin{tabular}{lllllllll} 
Jahr & $\begin{array}{l}\text { Kt. AG [7] } \\
\text { (EG HSK) }\end{array}$ & Kt. BE [8] & $\begin{array}{l}\text { Kt. BS [9] } \\
\text { (EG HSK) }\end{array}$ & $\begin{array}{l}\text { Kt. FR [10] } \\
\text { (EG HSK) }\end{array}$ & $\begin{array}{l}\text { Kt. SG [11] } \\
\text { (EG HSK) }\end{array}$ & Kt. SZ [12] & $\begin{array}{l}\text { Kt. VD [13] } \\
\text { (EG HSK) }\end{array}$ & $\begin{array}{l}\text { Kt. ZH [14] } \\
\text { (EG HSK) }\end{array}$ \\
\hline 2012 & 9931 & 9940 & 10028 & 9489 & 9580 & 9717 & 9756 & 9603 \\
\hline 2013 & 9902 & 9870 & 9893 & 9580 & 9700 & 9699 & 9756 & 9509 \\
\hline 2014 & 9856 & 9725 & 9738 & 9438 & 9630 & 9672 & 9650 & 9509 \\
\hline 2015 & 9666 & 9590 & 9690 & 9400 & 9564 & 9672 & 9640 & 9509 \\
\hline 2016 & $9400[15]$ & & & & & &
\end{tabular}

hängig davon, ob sie ambulant oder stationär erfolgen, abgegolten werden. Ist eine stationäre Behandlung erforderlich, soll der Mehraufwand verhältnismässig abgegolten werden.

\section{Problemkreis:}

\section{Abnahme der Entgeltung stationär erbrachter Leistungen}

Der Zunahme der Gesundheitskosten im stationären Bereich wird durch eine Reduktion des Basispreises (Baserate) im SwissDRG-Abgeltungssystem begegnet. Tabelle 1 zeigt dies anhand der Entwicklung der Baserate in acht Kantonen. Die abnehmende Entgeltung im DRG-System führt zur Herausforderung, dass bestimmte Operationen und Behandlungen stationär nicht mehr kostendeckend erbracht werden können.

Ein Spital hat in dieser Situation folgende Handlungsoptionen: Entweder werden unrentable stationäre Leistungen nicht mehr erbracht, oder sie werden, soweit medizinisch verantwortbar, in einem optimierten ambulanten Setting kostengünstiger erbracht. Eine Leistung ambulant statt stationär zu erbringen reduziert den Aufwand in personeller, infrastruktureller, logistischer und administrativer Hinsicht.

\section{Problemkreis: Grosser Investitionsbedarf}

Wegen Überalterung der Infrastruktur, Standortzusammenlegungen und Erweiterungsbauten besteht schweizweit grosser Investitionsbedarf, vor allem bei den öffentlichen Spitälern. In einer im August 2013 publizierten Studie zum Gesundheitswesen Schweiz hält die Credit Suisse fest: «Zurzeit sind Bauprojekte im Umfang von knapp 9 Mrd. CHF absehbar - der tatsächliche Investitionsbedarf der kommenden 15-20 Jahre dürfte jedoch noch einiges darüber liegen. Diesen Investitionsbedarf zu finanzieren und gleichzeitig die Kosten zu reduzieren, wird eine der grössten Herausforderungen des Spitals der Zukunft sein.» [16] Die Investitionskosten müssen von jedem Spital selbst getragen werden. Sie werden nicht mehr separat abgegolten, sondern sind in der Entgeltung der erbrachten Leistungen enthalten.

Es stellt sich demnach die Herausforderung, hohe Investitionen in die Infrastruktur zu tätigen und zu finanzieren - bei abnehmender Entgeltung (s. 2. Problemkreis) und bei laufendem Betrieb.

Die abnehmende Entgeltung im DRG-System führt dazu, dass bestimmte Operationen und Behandlungen stationär nicht mehr kostendeckend erbracht werden können.

Als sinnvolle Handlungsoption in dieser Situation erscheint eine differenzierte Investitionstätigkeit: Teurere Infrastruktur für die Erstellung stationärer Leistungen, günstigere Infrastruktur für die Erstellung ambulanter Leistungen.

\section{Problemkreis: Hohe Komplexität der Leistungserstellung}

Für den Ökonomen Peter Ulrich bedeutet Komplexität einer Situation die Vielfalt der einwirkenden Faktoren und das Ausmass ihrer gegenseitigen Interdependenzen. Komplexität ist das Merkmal schlecht strukturierbarer Entscheidungssituationen. Ihr Gegenteil ist Einfachheit, Determinierbarkeit, Überschaubarkeit [17].

Ein Spital zu führen und zu managen ist eine Aufgabe mit hoher Komplexität. Dies halten auch Tuckermann et al. in der dreiteiligen Artikelserie «Management von Spitälern - eine unmögliche Aufgabe?!» fest: «Nimmt man Spitäler als Organisationen näher in den Blick, kann man den Eindruck gewinnen, dass Management als Funktion dieser Organisationen eine schier unmögliche Aufgabe darstellt: [...] Zwischen organisationsweiten Fragestellungen und der Vielfalt von Professionen und Disziplinen entsteht ein 
Spannungsfeld, in dem Entscheidungen unmöglich oder vielmehr paradox werden: Die Vielfalt erfordert organisationsweite Entscheidungen, behindert sie aber zugleich.» [18] Eine zu hohe Komplexität bedeutet Gefahr: Die Vitalität eines Akteurs (beispielsweise eines Spitals) sinkt, wenn die Komplexität seiner Umwelt ansteigt [19]. Verschärfte Wettbewerbs- und Rahmenbedingungen, denen die Leistungserbringer im Schweizer Gesundheitswesen ausgesetzt sind, bedeuten zudem mehr Dynamik. Mehr Dynamik führt zu mehr Komplexität [20].

Mit Komplexität umgehen bzw. sie reduzieren zu können wird für medizinische Leistungserbringer in einem umkämpften Markt zu einer Herausforderung, die zu bewältigen essentiell ist.

Medizinische Leistungen in einem ambulanten statt in einem stationären Setting durchzuführen erfordert andere Prozesse, andere Strukturen und andere Denkhaltungen bei den Leistungserbringern. Werden ambulante und stationäre Leistungen in gleichen Prozessen und Strukturen und mit einer indifferenten Denkhaltung erbracht, steigt die Komplexität, und die Qualität der Leistung ist weniger als optimal. Wird hingegen die Leistungserstellung prozessual und infrastrukturell in einen stationären und in einen ambulanten Bereich aufgetrennt, nehmen die Zahl einwirkender Faktoren im jeweiligen Bereich und die gegenseitigen Wechselwirkungen ab. Die Auswirkungen des nicht vorhersehbaren Verhaltens autonomer Systemeinheiten werden geringer. Die Entscheidungssituationen werden strukturierbarer. Summa summarum: Die Komplexität wird reduziert. Eine Voraussetzung ist geschaffen, die Qualität der erbrachten Leistungen im ambulanten als auch im stationären Bereich deutlich zu erhöhen.

\section{Problemkreis: Fachkräftemangel}

Die Schweiz bildet selber nicht genügend Gesundheitsfachkräfte aus, um ihren Bedarf zu decken. Ihre attraktiven Arbeits- und Lebensbedingungen lösen dieses Problem: Fachkräfte aus dem Ausland kommen und decken den Bedarf. Dies ist allerdings unbefriedigend, u.a. hinsichtlich Versorgungssicherheit, Stabilität, Regenerationsfähigkeit und Wahrung der Eigenheiten des Gesundheitssystems. Effektiver wären Rahmenbedingungen, die die Fehlallokation von Fachpersonen im Gesundheitssystem reduzieren. Pflegepersonal und Ärzte sollten nicht bei Patienten, die dieser nicht bedürfen, eingesetzt werden.

Patienten im Spital übernachten zu lassen generiert insbesondere personellen Aufwand (pflegerisch, intellektuell, administrativ, emotional). Dieser Aufwand ist nicht nötig bei Behandlungen und Operationen, die ambulant durchgeführt werden können. Werden Patienten gleichentags wieder nach Hause entlassen, braucht es kein Personal für die Spät- und die Nachtschicht. Schnittstellen und damit auch Fehlerquellen fallen weg. Und den Patienten muss nicht erklärt werden, wieso sie im Spital übernachten, obschon dies offensichtlich fragwürdig ist. Solange die bestehenden finanziellen Fehlanreize [4] aber dazu führen, dass Patienten stationär behandelt werden, obschon sie keiner stationären Versorgung bedürfen, ist auch einer effektiven Bekämpfung des Fachkräftemangels der Weg versperrt.

Es stellt sich die Herausforderung, insbesondere die finanzielle Anreizsituation so zu verändern, dass medizinisches Fachpersonal nicht dort eingesetzt wird, wo sein Einsatz nicht erforderlich ist.

Eine Handlungsoption ist die bessere Abgeltung ambulant durchgeführter Behandlungen und Operationen. Eine bessere Abgeltung ist nicht nur relativ zu stationär erbrachten Leistungen angezeigt, sondern auch absolut. Damit eine Leistungserstellung im ambulanten Setting gelingt, ist ein höherer Aufwand erforderlich als unter stationären Bedingungen, denn

Die Abgeltung einer medizinischen Leistung sollte unabhängig davon erfolgen, ob sie ambulant oder stationär erbracht wird.

der Patient muss zwingend am gleichen Tag wieder nach Hause zurückkehren können. Beispielsweise bei einer Operation bedeutet dies: Die Durchführung der Anästhesie ist bezüglich Timing anspruchsvoller. Der postoperative Kontroll- und Interventionsaufwand ist höher, um einen ungünstigen Verlauf sofort zu erkennen und in die günstige Richtung zu steuern. Und die gute und nebenwirkungsarme postoperative Schmerzbehandlung ist anspruchsvoller und aufwendiger bezüglich Durchführung und Instruktion - sie muss zu Hause in jedem Fall gelingen. Dieser höhere Aufwand bei ambulanten Behandlungen und Operationen wird aktuell nicht adäquat abgegolten.

\section{«Wollen Sie ins Spital oder wollen Sie ambulant behandelt werden?»}

Die meisten Menschen bevorzugen die Annehmlichkeit, Zweckmässigkeit und Angemessenheit einer ambulanten Behandlung oder Operation. Sie schätzen die Vorteile, die die Vorbereitung und die Erholung 

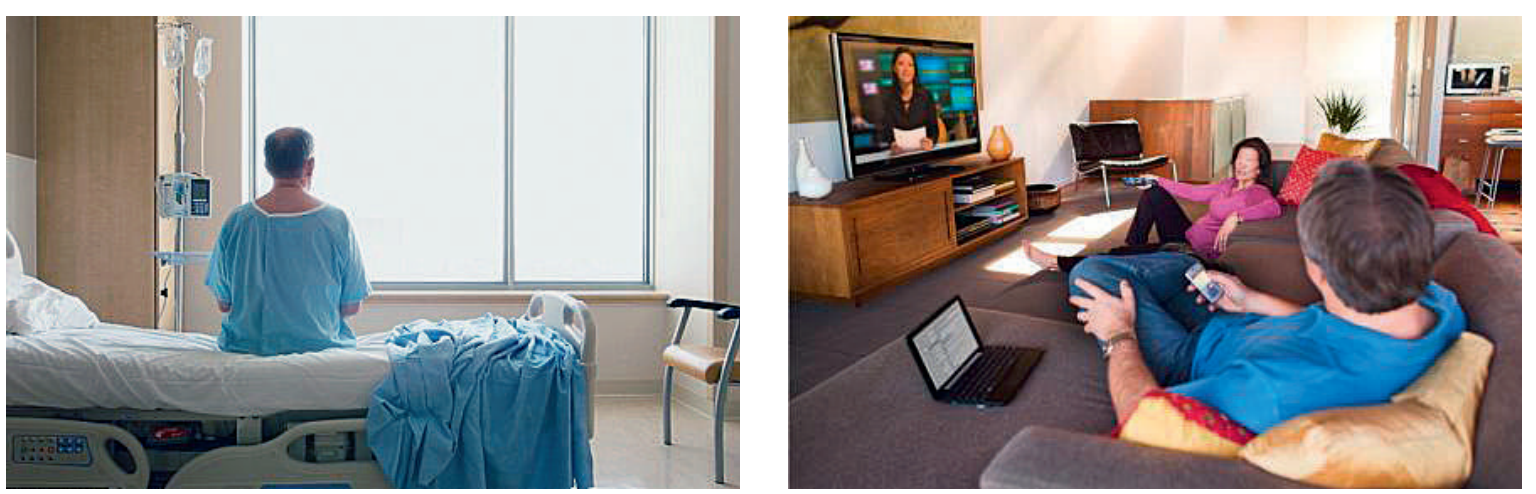

Menschen schätzen die Vorteile, welche die Vorbereitung und vor allem die Erholung von einem Eingriff bei sich zu Hause mit sich bringen.

von einem Eingriff bei sich zu Hause mit sich bringen [21]. Auch die verbreitete Angst, sich mit einem Spitalkeim zu infizieren, wird gegenstandslos.

\section{Es wird postuliert, dass mehr ambulante Behandlungen ein Gesundheitssystem patientenzentrierter und kundenfreundlicher machen.}

Im ambulanten Setting ist die Selbstbestimmung des Patienten grösser. Dies führt auch dazu, dass sein Einfluss auf den Betreuungsprozess allgegenwärtig ist. Es wird postuliert, dass mehr ambulante Behandlungen ein Gesundheitssystem patientenzentrierter und kundenfreundlicher machen. Mehr noch: Ambulante Behandlungen berücksichtigen die Mündigkeit, die eine moderne Gesellschaft kennzeichnet. Wer via Internet selber Bankgeschäfte tätigt, sich selber die Zugverbindung heraussucht und das Billett auf dem Smartphone löst und wer die Einkäufe an der Kasse selber scannt und bezahlt, der erwartet auch, bei der Behandlung seines gesundheitlichen Problems eine aktive und selbstbestimmte Rolle einnehmen zu können. Dies ist im ambulanten Setting eher möglich und für die Rekonvaleszenz sogar günstig.

\section{Den gordischen Knoten durchhauen}

Zusammenfassend haben mehr ambulant statt stationär erbrachte Leistungen substantielle Verbesserungen zur Folge:

- Das Kostenwachstum im Schweizer Gesundheitswesen nimmt ab.

Korrespondenz:

Dr. med. Michael Stamm

D.E.A.A.

Operationszentrum

Burgdorf AG

Farbweg 9

$\mathrm{CH}-3400$ Burgdorf

Tel. 03450808 o2

michael.stamm[at]

operationszentrum.ch mender Entgeltung im DRG-System weiterhin kostendeckend erbracht werden.

- Investitionen in medizinische Einrichtungen erfolgen differenzierter und kostengünstiger.

- Die Komplexität der Leistungserstellung nimmt ab und die Qualität nimmt zu.
- Dem Fachkräftemangel wird in effektiver Art begegnet.

Der Hebel für diese Verbesserungen ist die Abgeltung:

- Die Abgeltung einer medizinischen Leistung sollte unabhängig davon erfolgen, ob sie ambulant oder stationär erbracht wird (monistische Abgeltung) [22].

- Die Abgeltung des Mehraufwandes, den eine erforderliche stationäre Leistung mit sich bringt, sollte verhältnismässig ausfallen.

- Die Abgeltung ambulant erbrachter Leistungen sollte verbessert werden, sowohl relativ zur gleichen, stationär erbrachten Leistung, als auch absolut.

- Zusatzleistungen sollten auch im ambulanten Bereich zusätzlich abgegolten werden.

In ihrer Gesamtheit erinnert die aktuelle Situation im Schweizer Gesundheitswesen an einen gordischen Knoten, an eine scheinbar unlösbare Problemkonstellation. Der Knoten kann durchhauen werden, indem medizinische Leistungen wenn immer möglich standardmässig ambulant statt stationär erbracht werden. Dies ist medizinisch, wirtschaftlich und gesellschaftlich sinnvoll. Durch entschlossene Förderung ambulanter Behandlungen und Operationen würde das gesamte Schweizer Gesundheitswesen zahlreiche Impulse für eine günstige Weiterentwicklung und sogar einen Innovationsschub erhalten. Die spürbare Stagnation könnte überwunden werden.

Dank

Herzlichen Dank an Prof. Robert Leu für die kritische Gegenlektüre.
Interessenverbindungen

Der Autor ist medizinischer Leiter und Geschäftsführer der Operationszentrum Burgdorf AG. 


\section{Referenzen}

1 www.bfs.admin.ch/bfs/portal/de/index/news/o1/nip detail. html?gnpID $=2014$ - 095

2 www.bfs.admin.ch/bfs/portal/de/index/themen/14/o5/blank/ key/internationaler vergleich.html

3 www.bfs.admin.ch/bfs/portal/de/index/themen/o1/o2/blank/ key/alter/gesamt.html, Tabelle Struktur der ständigen Wohnbevölkerung, eigene Herleitung

4 Stamm M. Finanzielle Fehlanreize verhindern Kostensenkungen und Qualitätsverbesserungen. Schweiz Ärztezeitung. 2013;94(27-28): 1091-3. www.bullmed.ch/docs/saez/2013/2728/fr/ BMS-01617.pdf)

5 Woodtli N. Stationär statt ambulant: So kassieren Spitäler. Kassensturz 2014, Sendung vom 26.08.2014 (www.srf.ch/konsum/ themen/gesundheit/stationaer-statt-ambulant-so-kassierenspitaeler)

6 www.bfs.admin.ch/bfs/portal/de/index/news/ medienmitteilungen.html?pressID $=9434$

7 www.ag.ch/de/dgs/gesundheit/gesundheitsversorgung/ spitalfinanzierung/tarife_2/tarife_3.jsp

8 www.gef.be.ch/gef/de/index/gesundheit/gesundheit/ spitalversorgung/spitaeler/superprovisorischetarife.html

9 www.gesundheitsversorgung.bs.ch/gesundheitsfachpersonen/ spitalversorgung/spitalfinanzierung.html

10 www.fr.ch/ssp/de/pub/spitaeler/spitaeler.htm

11 www.sg.ch/home/gesundheit/gesundheitsversorgung/ spitaeler und kliniken/tarife stationaer.html

12 www.sz.ch/xml_1/internet/de/application/d5/d937/d610/d27237/ p27342.cfm

13 www.vd.ch/themes/sante/organisation/hopitaux-et-cliniques/ liste-lamal/prise-en-charge-des-couts/
14 www.gd.zh.ch/internet/gesundheitsdirektion/de/themen/ behoerden/spitalfinanzierung.html

15 Annahme gem. Berichterstattung des Projekts SMSB zur Planungserklärung des Grossen Rates vom 13.06.2013 (www.be.ch/portal/ de/index/mediencenter/medienmitteilungen.assetref/dam/ documents/portal/Medienmitteilungen/de/2014/o1/2014-1-14smsb-bericht-planungserkl\%C3\%A4rung.pdf)

16 Credit Suisse, Global Research: Gesundheitswesen Schweiz; 2013 August 2013, S4O.

17 http://de.wikipedia.org/wiki/Komplexit\%C3\%A4t

18 Tuckermann H, Rüegg-Stürm J, Mitterlechner M. Dreiteilige Artikelserie Management von Spitälern - eine unmögliche Aufgabe?! Schweizerische Ärztezeitung, Ausgaben 17, 18, 19/2014

19 Walter A. Das Schweizer Gesundheitswesen ist ein System. Schweiz Ärztezeitung. 2008;89(16):704-6.

20 Scholz-Reiter B, Tervo J. Kausaler Zusammenhang von Komplexität und Dynamik in der Produktion. In: Lucas K (Hrsg.). Kausalität in der Technik: Vorträge im Rahmen der wissenschaftlichen Sitzungen der Technikwissenschaftlichen Klasse (Akademie-Debatten), 2007; Berlin, S. 105-19 (http://edoc.bbaw. de/volltexte/2012/2278/)

21 Pomerantz P. Culture and Cost of Outpatient Care: Ambulatory Surgery and the Transformation of our Health System. ASA Newsletter 2013;77(7):10f.

22 Aussage von Urs Stoffel, Mitglied des Zentralvorstandes der FMH, in der Sendung Kassensturz vom 26.8.2014: «Wenn die Tarifpartner beispielsweise über Pauschalen verhandeln, bei denen es keine Rolle mehr spielt, ob eine Behandlung ambulant oder stationär erfolgt, ist die FMH sehr gerne bereit, in solche Verhandlungen einzusteigen.» 